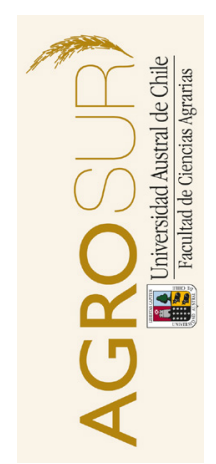

\begin{tabular}{l} 
A R T I C L E IN F O \\
\hline Article history: \\
Received 22.10.2018 \\
Accepted 27.12.2018 \\
\hline Keywords: \\
Ranunculaceae \\
Cut flower \\
Pulsing \\
Shelf life \\
Solution \\
Quality \\
\hline
\end{tabular}

Original Research Article, Food Science

${ }^{*}$ Corresponding author: Gabriela Verdugo-Ramírez E-mail address: gabriela.verdugo@pucv.cl

\section{Uso de soluciones azucaradas-ácidas de fácil formulación en pos cosecha de delfinio (Delphinium spp.)}

\author{
Use of sugar-acid solutions and easy formulation \\ in postharvest of Delphinium flower (Delphinium spp.)
}

\author{
Verdugo-Ramírez, G. ${ }^{a}{ }^{a} b *$, Vidal-Takasaki, A. ${ }^{a}$, Salgado-Varas, E. ${ }^{a, b}$ \\ ${ }^{a}$ Pontificia Universidad Católica de Valparaíso, Escuela de Agronomía. Casilla 4D Quillota, Chile. \\ ${ }^{b}$ AgerConsultores Ltda. Casilla 186 Quillota, Chile.
}

\begin{abstract}
A B S T R A C T
The use of different solutions in flower postharvest is based on substances of easy access for users and friendly to the environment. The main components of a postharvest solution correspond to sugars in an acid environment in such a way to reduce the proliferation of microorganisms that can alter the absorption and mobility of water. Using sugar-acid based solutions of fancy drinks can improve flower conditions in species with reduced postharvest life. In this experiment we tested solutions between 10 and $100 \%$ of the common soft drink Sprite $\AA$, because its favorable balance of sugar/acid. Results indicate that the control treatment onsets senescence on the 8th day after the start of experiment. In that moment treatments with more than $30 \%$ of Sprite $(\mathrm{pH}<3.6$ and sugar

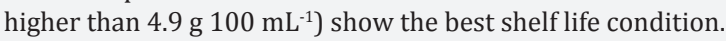

\title{
RESUMEN
}

El uso de diversas soluciones en pos cosecha de flores se basa en el empleo de sustancias de fácil acceso para los usuarios y amigables con el ambiente. Los componentes principales de una solución de pos cosecha corresponden a azúcares y se utiliza pH ácidos de tal modo de reducir la proliferación de microorganismos que pueden alterar la absorción y movilidad del agua. El uso de soluciones azucaradas ácidas en base a bebidas de fantasía puede mejorar las condiciones de las flores en especies de pos cosecha reducida. En estos ensayos se probó soluciones entre 10 y 100\% de Sprite. Los resultados indican que el tratamiento control inició la senescencia a los 8 días del inicio del experimento. En ese momento los tratamientos que incluían sobre $30 \%$ de Sprite ( $\mathrm{pH}<3,6$ y azúcar mayor que 4,9 g $100 \mathrm{~mL}^{-1}$ ) mostraron muy buena condición de vida de anaquel.

Palabras clave: Ranunculaceae, flor cortada, pulsado, vida de anaquel, solución, calidad.

\section{INTRODUCCIÓN}

Delfinio es una flor de corte interesante. El cultivo se inicia a partir de semilla, florece en forma de espiga, la que normalmente alcanza entre $70 \mathrm{~cm}$ a más de 1 $\mathrm{m}$ de altura, sus flores tienen colores principalmente azules, pero además se presenta en variedades blancas, crema y tonos rosados a lilas. Pertenece a la familia Ranunculaceae, y es una planta perenne de floración de verano y otoño. La flor tiene 5 sépalos falsos, los que a su vez contienen a los pétalos verdaderos (Wegulo y Vilchez, 2008; Porres, 2011; Arévalo et al., 2012).

Los índices de calidad de los delfinios incluyen, el largo del tallo, la rectitud del tallo, la calidad del follaje, el tamaño de las flores individuales por espiga y el porcentaje de apertura. La cosecha se hace con $1 / 3$ de la espiga mostrando color (Arévalo et al., 2012) o con 1 a 3 flores completamente abiertas. Tienen geotropismo negativo, por lo que se deben almacenar en posición vertical y dada su alta tasa de formación de etileno se recomienda almacenarlas entre 0 y $1{ }^{\circ} \mathrm{C}$ (Reid, 2013).

Es una interesante flor para arreglos voluminosos y aporta tonos inusuales en flores, que es un complemento buscado por los decoradores. No hay otras flores de características similares con las cuales compita. A pesar de todas estas buenas características hay poca producción, principalmente debido a las dificultades que esta especie presenta en pos cosecha. Reid (2013) 
la describe como una flor de pos cosecha difícil debido a que presenta alta sensibilidad al etileno y recomienda pre-tratar las espigas florales con 1-MCP o STS. Wagstaff et al. (2005) y Sánchez-Díaz et al. (2017) indican que el etileno es el coordinador de la senescencia en muchas flores, como también de otros órganos vegetales, y está presente en delfinio (Ichimura et al., 2000) y de allí la importancia de su control.

El uso de soluciones anti etileno tiene dificultades. El 1-MCP corresponde al metil ciclo propeno, desarrollado en 1999 en Estados Unidos, que a pesar de sus muy buenas cualidades como protector de flores cortadas (Verdugo et al., 2003; Verdugo et al., 2006), según Rademacher (2015) requiere instrucciones precisas para su uso en cada especie. Por otra parte, en algunos países no está inscrito para uso en flores de corte, entre ellos en Chile. El STS (tiosulfato de plata) debe ser formulado a partir de nitrato de plata y tiosulfato de sodio. El primer producto mencionado tiene detractores dado su efecto ambiental (López et al., 2008; Rademacher, 2015) y, en segundo lugar, requiere agua con bajo contenido de calcio para evitar precipitación durante su preparación, lo que en algunas zonas productivas puede ser una limitante. Por otra parte, el uso de soluciones caseras de diferentes bebidas cítricas como sodas de limón y lima (nombres comerciales: Sprite®, Seven Up ${ }^{\circledR}$, Limón Soda ${ }^{\circledR}$ ), y alcohólicas como vodka, solas o en mezcla con cloro, vinagre, jugo de manzana, enjuagues bucales entre otras, han sido probadas principalmente por las personas que se dedican a hacer o comercializar ramos de flores (Curtin, 2007; Rahman et al., 2012; Thwala et al., 2013).

Para reemplazar el uso de soluciones complejas (STS y 1-MCP) en la agricultura familiar se podría utilizar alguno de los tipos de soluciones caseras mencionadas. No obstante, no hay información científica que permita definir una combinación óptima de acidez y contenido de azúcar para prolongar la vida de anaquel de flores de delfinio. En consecuencia, el objetivo de este estudio es evaluar en delfinio el efecto de diversas concentraciones de acidez y azúcar, en tratamiento de pulsado, sobre tamaño de flor abierta, pérdida de peso, apertura de inflorescencia e índice de calidad.

\section{MATERIAL Y MÉTODOS}

Se realizó un ensayo preliminar en noviembre de 2015 con tallos de delfinio recién cortados, producidos en La Palma, Quillota (latitud $32^{\circ} 55^{\prime}$ S, longitud $71^{\circ} 20^{\prime}$ 0, altitud de 70 m s.n.m). Los tallos utilizados provinieron de plantas de dos años, producidas a partir de semilla, de la variedad Guardian, (BallChile $®$ ), homogenizadas por largo y estado de cosecha. Las flores fueron sometidas a tres tratamientos: (i) $600 \mathrm{cc}$ de Sprite (solución 100\%), (ii) solución de 300 cc de Sprite +300 cc de agua desionizada (solución 50\%) y (iii) 600 cc de solución de sacarosa $(0,1 \%)$ y ácido bórico $(0,1 \%)$. Luego de 24 horas, el $50 \%$ de los tallos de cada tratamiento se trasladó a recipientes con agua potable, y el resto se mantuvo en la solución inicial. Los tallos fueron puestos en dicha solución en una sala acondicionada según las indicaciones de Reid y Kofranek (1981) a $20 \pm 2{ }^{\circ} \mathrm{C}$, humedad relativa de $70 \pm 5 \%$ y 12 horas de luz a una intensidad PAR de 50 micromoles $\mathrm{cm}^{-2}$. El experimento se extendió hasta que una vara en un tratamiento mostró inicio de senescencia, lo que ocurrió al día 6 después de cortadas. En ese momento fueron evaluadas en una escala de calidad de 8 puntos, donde 0 era la peor flor y 8 la mejor (Cuadro 1).

En este primer ensayo se aplicó un diseño factorial completamente al azar en que los factores fueron: solución inicial (SI) y solución de mantenimiento (SM). Se realizaron 3 repeticiones de 6 tallos cada una. Posteriormente se realizó un segundo ensayo (abril de 2016) en el que se evaluaron las soluciones de Sprite $₫$ al 100 y 50\%, y además soluciones al 33, 25, 20, 10\%

Cuadro 1. Escala de evaluación aplicada a cada vara de delfinio.

Table 1. Assessment applied to each delphinium stem.

\begin{tabular}{ccccc}
\hline Puntaje & Tallo & Hojas & Crecimientos secundarios & Flor \\
\hline 8 & Verde & Hidratadas & Con desarrollo & Hidratadas y del color original \\
7 & Verde & Hidratadas & Con desarrollo & Ligera pérdida de color \\
6 & Verde & Hidratadas & Con desarrollo & Pérdida de color \\
5 & Verde & Hidratadas & Con desarrollo & Pérdida de color e hidratación \\
4 & Verde con sectores marrón & Algo deshidratadas & Sin desarrollo & Pérdida de color e hidratación \\
3 & Prioritariamente marrón & Algo deshidratadas & Sin desarrollo & Pérdida de color e hidratación \\
2 & Marrón completo & Deshidratadas & Sin desarrollo & Pérdida de color e hidratación \\
1 & Marrón y deshidratado & Deshidratada & Sin desarrollo & Deshidratada y descolorida \\
\hline
\end{tabular}


y un control con agua potable. En este segundo ensayo se caracterizó los tratamientos en base a pH medido de manera directa mediante un pHmetro ExStik II (Extech ${ }^{\circledR}$ Instrument, Taiwan) y el contenido de sacarosa en una alícuota de $40 \mathrm{ml}$ calculado en base al contenido indicado en el producto comercial Sprite (Cuadro 2).

El color inicial de las flores abiertas de ambos ensayos fue medido en colorímetro portátil (Precision Colorimeter NR10. CESMM, China) correspondiendo a base $\mathrm{L} 45,09 \mathrm{a}^{*} 16,44 \mathrm{y} \mathrm{b}^{*}-36,64$ y sobre color violeta $\mathrm{L} 40,77$ $a^{*} 39,55 b^{*}-45,52$.

Al igual que en el primer ensayo, pero esta vez después de 20 horas en la solución correspondiente a cada tratamiento, todas las flores fueron trasladadas a recipientes con agua potable (con una columna de $8 \mathrm{~cm}$ ).

El segundo ensayo se condujo en un diseño completo al azar, con 4 repeticiones de 6 tallos. El puntaje de calidad fue analizado mediante el test no paramétrico de Kruskal Wallis, $(p \geq 0,05)$ y de separación de medianas de Mood ( $p \geq 0,05)$, el análisis del resto de las variables se realizó mediante ANDEVA y, cuando correspondió, separación de medias por test de Tukey p $\geq 0,05$. Todos los resultados de los ensayos fueron analizados con el programa Minitab R13.

\section{RESULTADOS Y DISCUSIÓN}

El análisis estadístico realizado a los datos del primer ensayo indicó que no hay interacción entre las soluciones (SI) y (SM) y que la duración de la flor con calidad superior depende del nivel de la solución inicial a la que estuvieron expuestas durante el pulsado (Cuadro 3).

Cuando se usó Sprite® al 100\% como solución inicial, a las 24 horas los tallos presentaron un doblamiento a nivel medio de su altura, el cual a las 48 horas desaparece, así los tallos alcanzan 6 días de pos cosecha con muy buena calidad, alto porcentaje de flores abiertas

Cuadro 2. Caracterización química de tratamientos ensayo 2.

Table 2. Chemical characterization of treatments test 2.

\begin{tabular}{llc}
\hline Tratamiento & $\mathrm{pH}$ & $\begin{array}{c}\text { Contenido de azúcar } \\
\text { en g en } 100 \mathrm{ml}\end{array}$ \\
\hline Sprite $100 \%$ & 3,3 & 9,8 \\
Sprite $50 \%$ & 3,5 & 6,5 \\
Sprite $33 \%$ & 3,6 & 4,9 \\
Sprite $\quad 25 \%$ & 3,8 & 3,9 \\
Sprite $\quad 20 \%$ & 3,9 & 3,3 \\
Sprite $\quad 10 \%$ & 4,0 & 1,95 \\
Agua potable & 6,5 & 0,00 \\
\hline
\end{tabular}

Cuadro 3. Comparación la calidad de las flores reflejada en escala de notas obtenidas a los 6 días de puestas en florero (valor promedio de 3 repeticiones de 6 flores).

Table 3. Comparison the quality of flowers reflected in note obtained scale implementations vase 6 days (average value of 3 replicates of 6 flowers).

\begin{tabular}{lc}
\hline Tratamientos solución inicial & $\begin{array}{c}\text { Calificación en índice } \\
\text { de calidad: } 1 \text { mala; } \\
8 \text { muy buena. }\end{array}$ \\
\hline Sprite $100 \%$ & $8 \mathrm{a}^{1}$ \\
Sprite $50 \%$ & $8 \mathrm{a}$ \\
Ácido bórico 0,6 g + azúcar 0,6 g L ${ }^{-1}$ & $3 \mathrm{~b}$ \\
P & 0,003 \\
\hline
\end{tabular}

${ }^{1}$ Promedios seguidos de distinta letra son estadísticamente diferentes según Kruskal-Wallis $\mathrm{p} \geq 0,05$.

en las espigas, desarrollo de brotes secundarios, tallos verdes e hidratados, flores hidratadas que permanecieron con el color inicial o con leves cambios (Figura 1), estos antecedentes indican una buena duración para la especie. Peck (2016) describe en sus ensayos con las mismas variedades duraciones 5,5 a 6,3 días. Se aprecia un incremento del color violeta por sobre el azul, pero sin llegar a afectar la apariencia, con diferencias de valores menores a 5 puntos en valor compuesto de luminosidad (L) a* y b* que según Velasco et al. (2017) no es perceptible. En tanto, el tratamiento de sacarosa y ácido bórico resulta en la pérdida de la calidad comercial en este mismo lapso.

En el segundo ensayo se usó flores formadas en otoño y naturalmente tienen mayor duración que aquellas formadas en períodos de calor y exceso de luz como primavera y verano, como fue probado en calas por Verdugo y Cueva (2008) y analizado por Ahmad et al. (2017). A los 8 días después de cortados los tallos se inició la senescencia, la cual se manifestó solamente con caída de pétalos de 3 flores individuales. En ese momento se registró la apariencia general, tamaño y peso de las flores, el porcentaje de apertura y la mantención del color (Figura 2).

Se alcanza un diámetro de flor abierta entre 4 y $6 \mathrm{~cm}$ siendo influido por la presencia de azúcar y $\mathrm{pH}$ ácido. Respecto a la pérdida de peso, que en general no supera el 5\% del peso inicial de los tallos, la mayor pérdida se observa en los tallos expuestos a las mayores concentraciones de azúcar y aquellos tratamientos que produjeron las flores de mayor diámetro. El hecho que las mayores pérdidas se observaron en los tratamientos con flores más grandes corrobora que las partes de las flores (pétalos, sépalos), son un importante elemento en la vida de una flor cortada (Ichimura et al., 2000). 


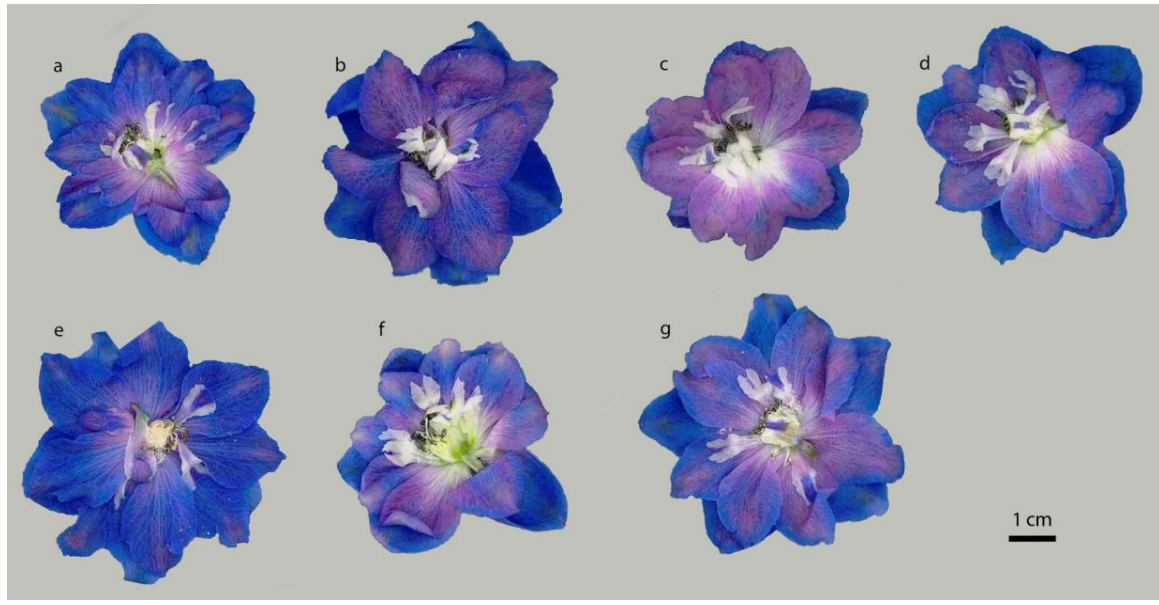

Figura 1. Estado de las flores individuales, tercera flor desde la base de la espiga, cosecha de los tallos con flores cerradas. Evaluación hecha a 8 días del tratamiento de soluciones de pulsado: a) control pH 6,5 y 0 g azúcar; b) pH 3,3 y 9,8 g azúcar; c) pH 3,5 y 6,5 g azúcar; d) pH 3,6 y 4,9 g azúcar; e) pH 3,8 y 3,9 g azúcar; f) pH 3,9 y 3,3; y g) pH 4,0 y 1,95 g azúcar.

Figure 1. State of individual flowers, third flower from the base of the stem, harvested stalks with closed flowers. Evaluation 8 days after treatment of pulsing solutions: a) control pH 6.5 and 0 g sugar; b) pH 3.3 and 9.8 g sugar; c) pH 3.5 and 6.5 g sugar; d) pH 3.6 and 4.9 g sugar; e) pH 3.8 and 3.9 g sugar; f) pH 3.9 and 3.3 g of sugar; and g) pH 4.0 and 1.95 g sugar.
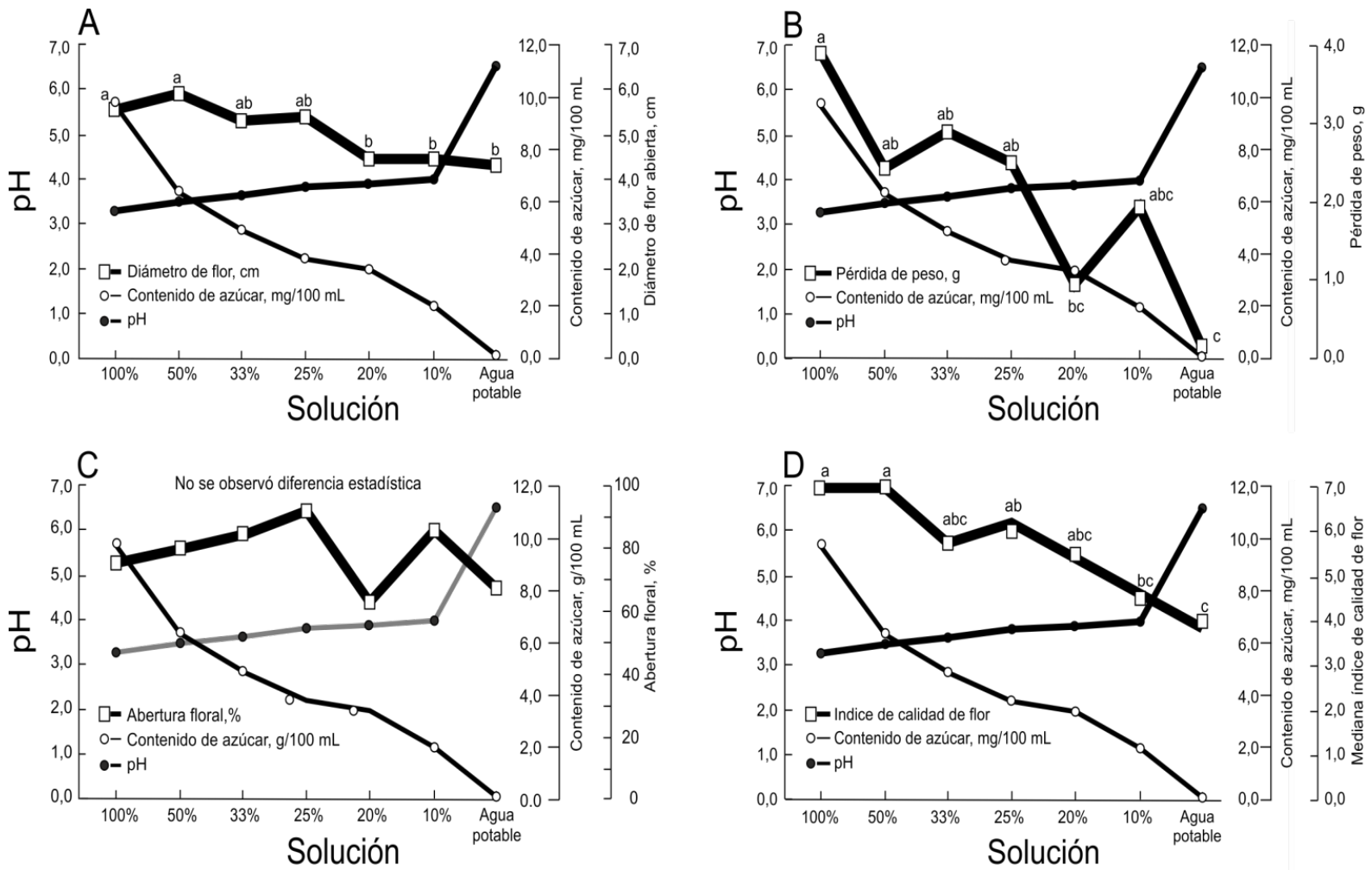

Figura 2. Efecto de los tratamientos en: A, el diámetro de la flor abierta (cm); B, en el peso de los tallos (g); C, apertura de la inflorescencia (\%); y D, la apariencia general de la flor (Escala de 1 a 8, mediana analizada mediante test no paramétrico Kruskal-Wallis $\mathrm{p} \geq 0,05$ y separación de medianas por Mood). Letras iguales en el gráfico, indican que no se encontraron diferencias entre los tratamientos. Valor promedio de 4 repeticiones de 6 tallos.

Figure 2. Treatment effects on: A, Open flower diameter (cm); B, Stem weight drop (g); C, Floral opening (\%); Floral quality index (scale 1 to 8, analyzed with Kruskal-Wallis and Mood). Same letter within the graph indicate that no differences among treatments were found. Mean value of 4 replicates of 6 stems. 
Hay dos factores que actúan en el resultado obtenido, por una parte, el $\mathrm{pH}$, observándose una variación entre las respuestas de tallos sometidos a una solución de $\mathrm{pH} 3,3$, y aquellas sometidas a $\mathrm{pH} 3,9$, y con la solución control en la que esa variable llegó a 6,5 y en la cantidad de azúcar, como se evidencia en el Cuadro 2. Las soluciones evaluadas mantienen un medio ácido que tiene como efecto principal bajar la tasa de multiplicación de microorganismos, y por ende, retarda el efecto taponamiento de haces conductores que impide el ascenso de agua como fue demostrado por Van Doorn et al. (1990). Por otra parte, en la medida en que se diluyó la cantidad de Sprite en las soluciones, disminuye el contenido de azúcar de las mismas, elemento que ha probado ser un sustrato de mantención de la actividad metabólica de los tallos cortadas aun cuando incremente la posibilidad de desarrollo de microorganismos (Kolar et al., 2017). Hay abundante literatura que respalda el uso de $\mathrm{pH}$ ácidos y azúcar en la pos cosecha de flores (Nowak y Rudnicki, 1990). Es conocido que la proliferación de bacterias en el agua del florero acorta la vida de las flores cortadas (Liao et al., 2001), y a pH más bajos se observa que las bacterias no proliferan (Tormo y Rochina, 2009). El efecto de la sacarosa (azúcar) en la prolongación de la vida pos cosecha de varias flores de corte ya ha sido demostrado (ShimizuYumoto y Ichimura, 2007; Ichimura et al., 2008).

\section{CONCLUSIONES}

La información recopilada en el presente estudio permite definir las concentraciones, $y$, por lo tanto, el contenido de azúcar y pH de las soluciones. Ésta respalda que el uso de Sprite® en solución al 33\% (solución de pH 3,8 y 3,9 g de azúcar por $100 \mathrm{ml}$ ) sirve para extender la vida útil de las flores en pos cosecha, llegando a su máximo potencial genético, descrito para híbridos de esta especie por Janssen (2002) entre 6 y 8 días. Sería recomendable su uso por los floricultores, aplicándola a las flores cortadas en forma de pulsado por 20 a 24 horas, para posteriormente trasladar los ramos a cubetas con $8 \mathrm{~cm}$ de altura de agua potable.

A contar del año 2018 las bebidas de fantasía fueron rebajadas en contenido de azúcar, por lo tanto hay que verificar el contenido real y llevarlo a 3,9 g de azúcar por L de agua destilada usada.

\section{REFERENCIAS}

Ahmad, I., Rafiq, M., Dole, J.M., Abdulah, B., Habib, K., 2017. Production and postharvest evaluation of selected exotic specialty annual cut flower species in Punjab, Pakistan. HortTechnology 27(6), 878-883.

Arévalo, L.A., Porres, M.A., De León, E., 2012. Evaluación de la adaptación de cuatro especies de flores de corte en cuatro localidades del departamento de Solalá. Revista 24 de la Universidad del Valle de Guatemala, 105-111.
Curtin, C., 2007. Fact or Fiction? Vodka and citrus sodas keep cut flowers fresh. Scientific American. https://www. scientificamerican.com/article/fact-or-fiction-vodkacitrus-sodas-keep-flowers-fresh/ (acceso, 08.06.2015).

Ichimura, K., Kohata, K., Goto, R., 2000. Soluble carbohydrates in Delphinium and their influence on sepal abscission in cut flowers. Physiologia Plantarum 108(3), 307-313. https://doi.org/10.1034/j.13993054.2000.108003307.x

Ichimura, K., Yoshioka, S., Yumoto-Shimizu, H., 2008. Effects of silver thiosulfate complex (STS) sucrose and combined pulse treatments on the vase life of cut snapdragon flower. Environmental Control in Biology 46(3), 155162. https://doi.org/10.2525/ecb.46.155

Janssen, D., 2002. Delphiniums - A Spectacular Garden Flower. University of Nebraska. http://lancaster.unl.edu/hort/ articles/2002/delphinium.shtml. (acceso, 10.09.2015).

Kolar, F.R., Sandeep, R.P., Ghansham, B.D., 2017. Delphinium malabaricum (huth) munz: a potential ornamental crop from Western Ghats. Horticulture International Journal 1 (1), 16-21. https://doi.org/10.15406/ hij.2017.01.00003

Liao, L.J., Lin, Y.H., Huang, K.L., Chen, W.S., 2001. Vase life of Eustoma grandiflorum as affected by aluminum sulfate. Botanical Bulletin Academia Sinica 42(1), 35-38.

López, P., Neisa, D.P., Bacca, C., Flórez, V.J., 2008. Evaluación de preservantes florales en la poscosecha de tres variedades de clavel estándar. Agronomía Colombiana 26(1), 116-126.

Nowak, J., Rudnicki, R.M., 1990. Postharvest handling and storage of cut flowers, florist greens, and potted plants. Springer Netherlands, Timber Press, Inc., London, England.

Peck, L.K., 2016. Pre- and Postharvest Practices for Optimizing the Postharvest Quality of Cut Delphinium, Dahlia, and Sunflower. Master thesis in Horticulture. Virginia Tech Electronic theses and Dissertations. https://vtechworks.lib.vt.edu/handle/10919/73589

Porres, M.A., 2011. Evaluación de cinco especies de flores de corte bajo infraestructura mínima y a campo abierto en cuatro localidades de Sololá. Fondecyt 033-2008 Universidad del Valle de Guatemala. Ciudad de Guatemala, Guatemala. 48 p.

Rademacher, W., 2015. Plant growth regulators: backgrounds and uses in plant production. Journal of Plant Growth Regulation 34(4), 845-872. https://doi.org/10.1007/ s00344-015-9541-6

Rahman, M.M., Ahmad, S.H., Lgu, K.S., 2012. Psidium guajava and Piper betle leaf extracts prolong vase life of cut carnation (Dianthus caryophyllus) flowers. The Scientific Word Journal 2012, 1-9. https://doi. org/10.1100/2012/102805

Reid, M., 2013. Delphinium: recommendation for maintaining postharvest quality. http://postharvest.ucdavis. edu/Commodity_Resources/Fact_Sheets/Datastores/Ornamentals_English/?uid=13\&ds=801 (acceso, 15.10.2015).

Reid, M.S., Kofranek, A.M., 1981. Recommendations for standardized vase life evaluations. Acta Horticulturae 113, 171-174. https://doi.org/10.17660/ActaHortic.1981.113.25

Sánchez-Díaz, J.M., Jiménez-Becker, S., Jamilena, M., 
2017. A screening test for the determination of cut flower longevity and ethylene sensitivity of carnation. Horticultural Science 44(1), 14-20. https://doi. org/10.17221/134/2015-HORTSCI

Shimizu-Yumoto, H., Ichimura, K., 2007. Effect of relative humidity and sucrose concentration of leaf injury and vase life during sucrose pulse treatment in cut Eustoma flowers. Horticultural Research (Japan) 6(2), 301-305. https://doi.org/10.2503/hrj.6.301

Thwala, M., Wahome, P.K., Oseni, T.O., Masarirambi, M.T., 2013. Effects of floral preservatives on the vase life of orchid (Epidendrum radicans L.) cut flower. Journal of Horticultural Science \& Ornamental Plants 5(1), 22-29. https://doi.org/10.5829/idosi.jhsop.2013.5.1.268

Tormo, V., Rochina, I.J., 2009. Antisépticos: fundamentos de uso en la práctica clínica. Ed. V. Tormo. La Eliana, Valencia, España.

Velasco, V., Bravo, P., Williams, P., Campos, J., Astudillo, R., Melín, P., 2017. Estabilidad durante el almacenamiento de carne de pollos alimentados con orégano seco (Origanum vulgare L.) en la dieta. Chilean Journal of Agricultural \& Animal Sciences 33(1), 28-38. http://dx.doi. org/10.4067/S0719-38902017005000104

Verdugo, G., Cueva, R., 2008. Estado de cosecha y mantención en cámara de calas Green Goddess y Red Desiré. Horticultura Revista de Industria Distribución y Socioeconomía Hortícola 208, 26-29.

Verdugo, G., Biggi, M., Montesinos, A., Soriano, C., Chahin, G., 2006. Manual de poscosecha de flores cortadas. Pontificia Universidad Católica de Valparaíso - Fundación para la Innovación Agraria, Valparaíso, Chile, 79 p.

Verdugo, G., Araneda, L., Riffo, M.O., 2003. Efecto de inhibidores de etileno en postcosecha de flores cortadas de Lilium. Ciencia e Investigación Agraria 30(2), 89-95.

Van Doorn, W.G., deWitte,Y.,Perik, R.R.J., 1990. Effect of antimicrobial compounds on the number of bacteria in stems of cut rose flowers. Journal of Applied Bacteriology 68(2), 117-122. https://doi.org/10.1111/j.1365-2672.1990. tb02555.x

Wagstaff, C., Chanasut, U., Harren, F.J.M., Laarhoven, L., Thomas, B., Rogers, H.J., Stead, A.D., 2005. Ethylene and flower longevity in Alstroemeria: relationship between tepal senescence, abscission and ethylene biosynthesis. Journal of Experimental Botany 56(413), 1007-1016. https://doi.org/10.1093/jxb/eri094

Wegulo, S.N., Vilchez, M., 2008. Evaluation of delphinium cultivars for resistance to powdery mildew. HorTechnology 18(3), 407-409. 